\title{
INFLUÊNCIA DA ADIÇÃO DE TENSOATIVO NO TAMANHO DE BOLHAS E HOLDUP DE AR NA FLOTAÇÃO EM COLUNA
}

\author{
A. M. R. FILHO ${ }^{1}$, G. R. L. e CARVALHO ${ }^{1}$, P. H. M. LUZ ${ }^{1}$, A. S. REIS ${ }^{1}$, M. A. S. \\ BARROZO $^{1}$ \\ ${ }^{1}$ Universidade Federal de Uberlândia, Faculdade de Engenharia Química \\ E-mail para contato: sreis.angelica@gmail.com
}

\begin{abstract}
RESUMO - No beneficiamento de minérios, como a apatita, para promover a separação das partículas de interesse dos minerais de ganga, um dos métodos utilizados é a flotação em coluna. A flotação é um processo de concentração baseado no comportamento físico-químico das superfícies das partículas presentes em uma suspensão aquosa. Visto que o diâmetro de bolha é uma variável importante na otimização desse processo, a determinação e controle do tamanho das bolhas formadas tornam-se essenciais para caracterizar o processo de flotação e, assim, determinar os valores operacionais que resultem em melhores recuperações da apatita. A utilização de tensoativos no processo de flotação tem como principais funções controlar o tamanho de bolha, produzindo bolhas menores aumentando assim a probabilidade de colisão bolha-partícula e consequentemente o aumento do holdup de ar. Desta forma este trabalho teve como objetivo avaliar a influência da adição de tensoativo observando as variações do tamanho de bolha e do holdup de ar. Para isso foram realizados testes nos quais foram variáveis a velocidade superficial do gás, concentração de tensoativo e a vazão de alimentação de tensoativo. Para determinar o diâmetro de bolhas foram utilizadas técnicas de filmagem e o holdup foi calculado através de medidas obtidas em manômetros diferenciais. Os resultados mostraram que ao longo do tempo o tensoativo agiu diminuindo o tamanho de bolhas juntamente com o aumento do holdup conforme visto na literatura. Conclui-se que a utilização de tensoativo é eficaz para geração de bolhas menores em coluna de flotação.
\end{abstract}

\section{INTRODUÇÃO}

A flotação em coluna é um processo físico-químico utilizado no beneficiamento de minérios, como a apatita, com o objetivo de promover a separação das partículas de interesse dos minerais de ganga. Nesse processo, bolhas de ar são injetadas e/ou formadas no interior da coluna. Assim, as partículas hidrofóbicas se aderem à superfície das bolhas formando agregados bolha-partículas que ascendem (flotam), formando uma fase de espuma coletada no topo do equipamento. As partículas hidrofílicas permanecem em suspensão aquosa e são removidas na base do equipamento, como rejeito.

Visto que o diâmetro de bolha é uma variável importante na otimização desse processo, a determinação e controle do tamanho das bolhas formadas tornam-se essenciais para 
caracterizar o processo de flotação e, assim, determinar os valores operacionais que resultem em melhores recuperações do mineral de interesse.

Vários estudos são realizados para o controle do tamanho de bolha, sendo o uso de tensoativos uma das alternativas. Em geral, a estrutura molecular dos tensoativos (espumantes) apresenta uma parte hidrofóbica (não polar) e uma hidrofílica (polar). Comumente a parte hidrofílica é representada pelo grupo $\mathrm{OH}$ (como no caso dos alcoóis) e, a parte hidrofóbica é uma cadeia hidrocarbônica. Devido a sua bipolaridade, o espumante adsorve na superfície da bolha de maneira a ficar com a parte hidrofílica voltada para a água e a hidrofóbica para a bolha (FINCH et al., 2008).

A utilização de tensoativos no processo de flotação tem como principais funções controlar o tamanho de bolha, produzindo bolhas menores aumentando assim a probabilidade de colisão bolha-partícula, e prover a estabilização da espuma (CHO E LASKOWSKI, 2002; FINCH et al., 2008; SOBHY e TAO, 2013; SANTOS, 2014).

Diante da necessidade de controle do tamanho de bolhas para aumentar a probabilidade de colisão bolha-partícula e, consequentemente, a recuperação do processo de flotação, este trabalho teve como objetivo verificar a influência da adição de tensoativo no diâmetro de bolha e no holdup de ar ao longo do tempo.

\section{Materiais e Métodos}

Unidade Experimental: A unidade experimental, representada pela Figura 1, encontra-se no Laboratório de Sistemas Particulados da Faculdade de Engenharia Química da Universidade Federal de Uberlândia. A unidade consiste de uma coluna de flotação feita de acrílico composta por uma seção cilíndrica com $150 \mathrm{~cm}$ de comprimento de $4 \mathrm{~cm}$ de diâmetro, abaixo uma seção tronco-cônica com $9,5 \mathrm{~cm}$ de altura e abaixo desta seção cilíndrica com 12 $\mathrm{cm}$ de altura e $10 \mathrm{~cm}$ de diâmetro.

Figura 1 - Unidade Experimental

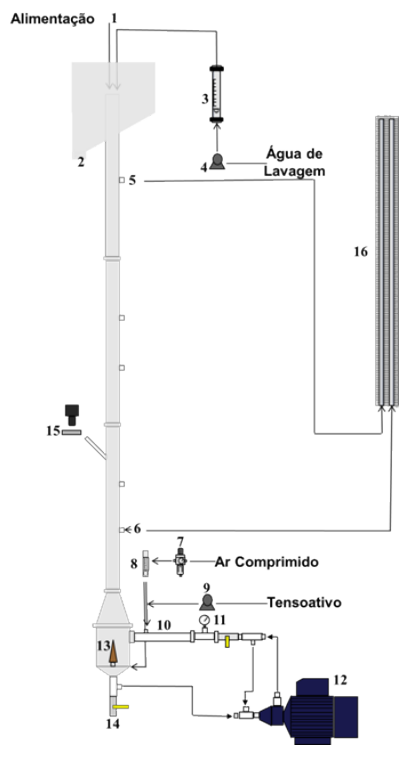

Legenda:

1 - alimentação de água

2 - ponto de coleta do

concentrado

$3,4,5,6,7$ - pontos de

amostragem do holdup

8 - sistema de alimentação de ar

comprimido com filtro de ar e

rotâmetro

9 - Venturi

10 - aerador

11 - bomba peristáltica

12 - ponto de coleta do rejeito

13 - sistema de amostragem de

bolhas

14 - manômetros diferenciais 
Metodologia para a determinação do holdup: O valor foi determinado através dos manômetros diferenciais (16) ilustrados na Figura 1. A diferença de nível entre os manômetros era obtida quando não se observava uma variação significativa no holdup da coluna e daí se calculava o holdup de ar. Assim o holdup foi calculado como a razão entre o valor obtido entre do desnível entre os manômetros diferenciais $(\Delta \mathrm{h})$ pela distância entres ospontos de amostragem(L).

$\varepsilon_{0}=\frac{\Delta h}{L}$

Metodologia para determinação do diâmetro das bolhas: Para a medida do diâmetro das bolhas foram utilizadas duas técnicas de filmagem realizadas com o auxílio de uma câmera de alta velocidade e um estereomicroscópio. Uma das técnicas consistia na amostragem de bolhas de forma intrusiva e a segunda a filmagem direta das bolhas foi realizada de maneira não-intrusiva. Uma melhor descrição dessas técnicas podem ser obtidas no trabalho realizado por Filho et al. (2014).

\section{Planejamento Experimental}

Com o objetivo de conhecer o comportamento do sistema com a adição de tensoativo e determinar qual a faixa de valores a ser utilizada para cada variável, foram realizados três testes com o sistema operando com condições máxima, intermediária e mínima das variáveis.

As variáveis estudadas foram a velocidade superficial do gás $\left(\mathrm{J}_{\mathrm{G}}\right)$, a concentração de tensoativo (C), a vazão de alimentação do tensoativo $\left(\mathrm{Q}_{t}\right)$. Os detalhes dos testes são mostrados na Tabela 1.Nestes testes, a pressão de ar foi fixada em 3 bar e as amostragens foram realizadas a cada $3 \mathrm{~min}$ durante o tempo total de $24 \mathrm{~min}$. Foi utilizado o Genagen, como tensoativo.

Tabela 1 - Testes

\begin{tabular}{c|c|c|c}
\hline Teste & $\begin{array}{c}\mathbf{J}_{\mathbf{G}} \\
(\mathbf{c m} / \mathbf{s})\end{array}$ & $\begin{array}{c}\mathbf{C} \\
(\mathbf{p p m})\end{array}$ & $\begin{array}{c}\mathbf{Q}_{\mathbf{t}} \\
(\mathbf{m L} / \mathbf{m i n})\end{array}$ \\
\hline $\mathbf{1}$ & 2,5 & 50 & 100 \\
\hline $\mathbf{2}$ & 1,5 & 30 & 50 \\
\hline $\mathbf{3}$ & 0,5 & 5 & 14 \\
\hline
\end{tabular}

Dessa forma, a partir da Equação 2, foi calculada a velocidade superficial do gás $\left(\mathrm{J}_{\mathrm{G}}\right)$, variável escolhida para ser analisada nos próximos planejamentos, visto que ela tem sido mais utilizada em vários trabalhos científicos. Outro motivo é o fato de que é uma referência para os processos de flotação, da qual muitos afirmam que uma boa eficiência no processo de flotação ocorre para $J_{\mathrm{G}}$ na faixa de $1-2 \mathrm{~cm} / \mathrm{s}$.

$\mathrm{J}_{\mathrm{G}}=\frac{\mathrm{Q}_{\mathrm{ar}}}{\mathrm{A}}$ 


\section{Resultados e discussão}

A ação do tensoativo, no diâmetro de bolha e no holdup, ao longo do tempo pode ser observada através da Figura 2. A Figura 2-a mostra que, como mencionado na literatura por vários autores (SHUKLA et al, 2010; LEIVA et al., 2010; GRAU et al., 2005; ALDRICH E FENG, 2000), o tensoativo atua de forma eficaz na diminuição do tamanho de bolha, ou melhor, sua presença no sistema diminuiu a ocorrência do fenômeno de coalescência, mantendo assim, as bolhas com diâmetros menores.

Observa-se também que a ação do tensoativo sobre o diâmetro de bolha tende a um valor fixo, mostrando que há uma estabilização do sistema. O tensoativo adicionado em um tempo maior atua apenas para manter o tamanho das bolhas.

Na Figura 2-b é possível notar que a adição de tensoativo também agiu de maneira efetiva no valor do holdup $\left(\varepsilon_{\mathrm{o}}\right)$, tendo como consequência um aumento do holdup à medida com que se passava o tempo e o tensoativo era adicionado. Observa-se que o aumento do $\varepsilon_{0}$ tende a estabilizar, da mesma forma como aconteceu para o $\mathrm{D}_{32}$, o que era esperado, já que existe uma relação direta entre o tamanho de bolha e o holdup.

Figura 2 - Influência ao longo do tempo da adição de tensoativo no $\mathrm{D}_{32}$ e no holdup.

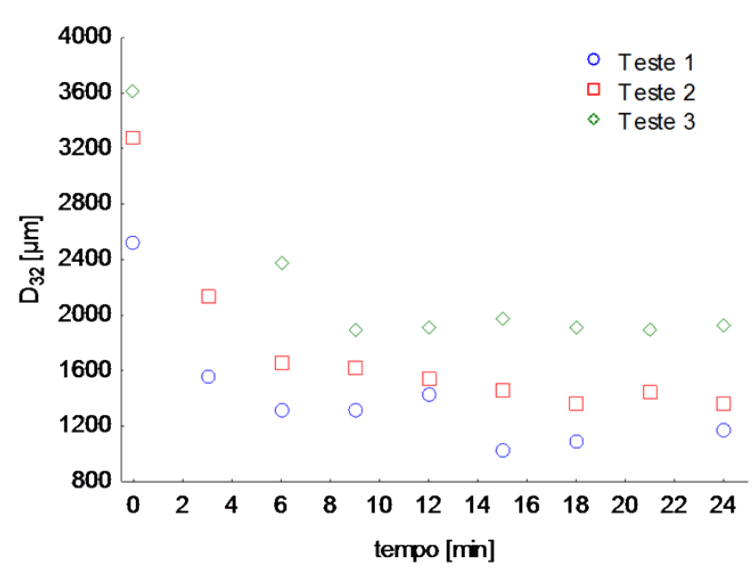

$\mathrm{a}$

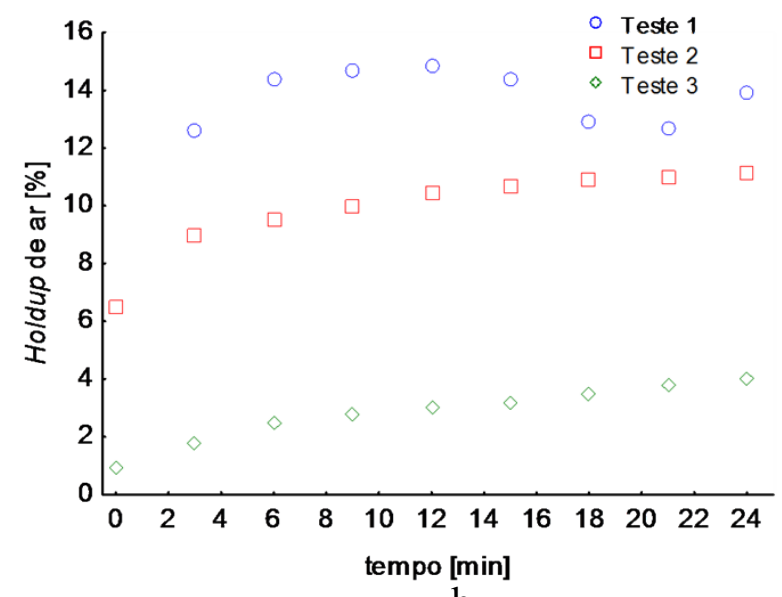

b

A partir destes resultados, foi possível observar que no Teste 3 , no qual foram utilizadas as menores condições para vazão de ar, concentração e alimentação de tensoativo, obteve-se uma diminuição de bolha, relativa ao tamanho inicial, próxima com os outros testes. Porém, mesmo com o aumento provocado pelo tensoativo, o valor do holdup continuou baixo comparado com o desejado (5-35\%) e as bolhas continuaram grandes. Portanto, definiu-se a concentração de 5 ppm de tensoativo como o valor mínimo para os testes posteriores a esse.

A mesma análise foi feita para o Teste 1, no qual a vazão de ar, concentração e alimentação de tensoativo foram testadas com valores mais altos. Notou-se que essas condições foram suficientes para diminuir o diâmetro médio das bolhas a valores dentro da faixa de bolhas com tamanho intermediário. Com relação ao holdup, verificou-se que houve 
um aumento significativo alcançando o valor de $15 \%$, valor médio na zona de recuperação da faixa especificada como favorável para o processo de flotação.

A Figura 3 mostra uma sequência de fotos, obtidas no método da amostragem de bolhas, relativas a cada um dos tempos amostrados. Essas fotos comprovam o efeito do tensoativo observado pelos gráficos, nas quais foi possível observar a diminuição do tamanho das bolhas e o aumento na concentração de bolhas, que diz respeito ao aumento do $\varepsilon_{0}$.

Figura 3: Fotos mostrando a ação do tensoativo no tamanho de bolha $\left(\mathrm{D}_{32}\right)$ e no holdup $\left(\varepsilon_{\mathrm{o}}\right)$.

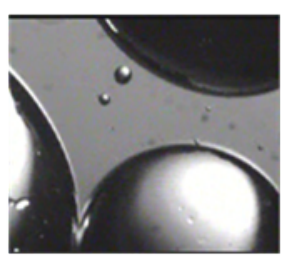

$\mathrm{t}=0 \mathrm{~min}$

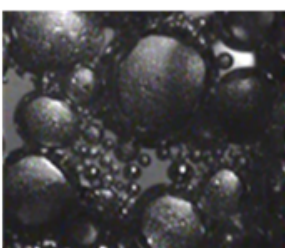

$\mathrm{t}=12 \mathrm{~min}$

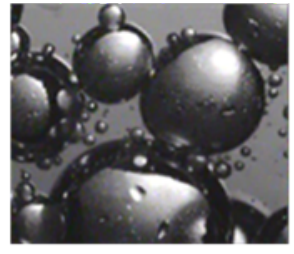

$\mathrm{t}=3 \mathrm{~min}$

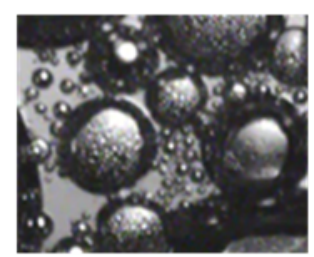

$\mathrm{t}=18 \mathrm{~min}$

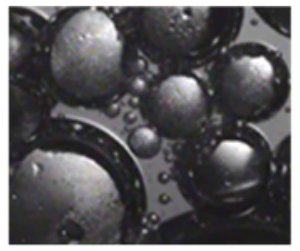

$\mathrm{t}=6 \mathrm{~min}$

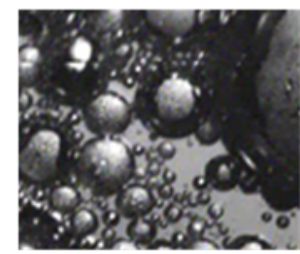

$\mathrm{t}=21 \mathrm{~min}$

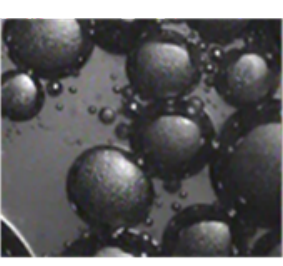

$\mathrm{t}=9 \mathrm{~min}$

Na Figura 4 é mostrado um histograma comparativo das DTB para o sistema ar-água sem e com a adição de tensoativo (Genagen). Nesse, fica visualmente evidente o efeito na diminuição do tamanho de bolhas, provocada pela adição do tensoativo.

Figura 4: Histograma comparativo das DTB do sistema ar-água operando sem e com a adição de tensoativo.

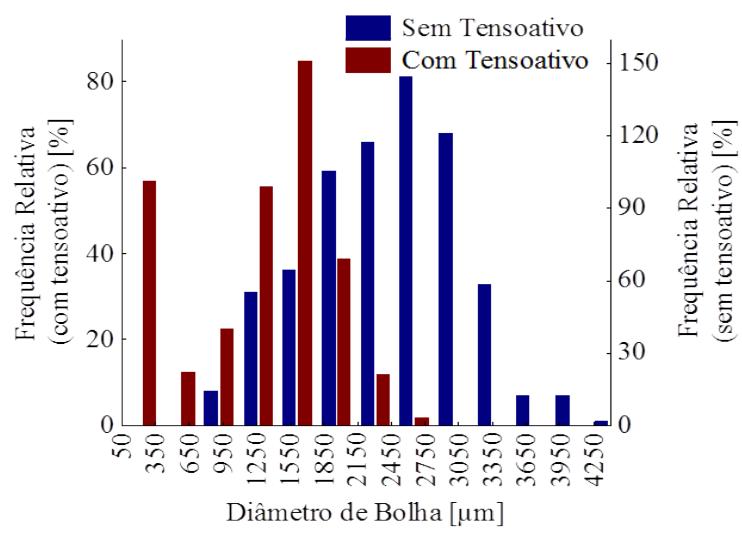

\section{Conclusões}

Conclui-se através dos resultados que o uso do tensoativo foi eficaz para a diminuição do diâmetro de bolha e o aumento do holdup de ar nos testes 1 e 2 que possuíam maior 


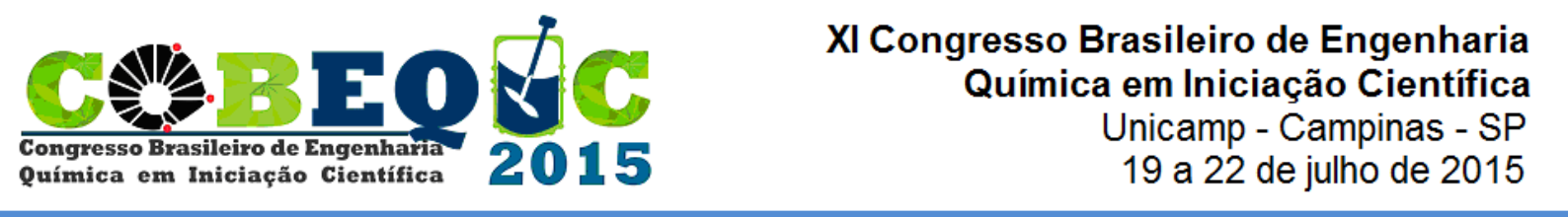

valores para as variáveis analisadas. Já no teste 3 , o uso de tensoativo acarretou a diminuição do diâmetro de bolhas mas não teve aumento significativo no holdup de ar, pois as bolhas ainda continuaram grandes devido a baixa concentração do tensoativo. Em todos os testes observou-se que ao passar do tempo ocorre uma estabilização do sistema mantendo fixo o tamanho de bolha. A DTB foi menor quando o sistema operou com adição de tensoativo de acordo com o previsto pela literatura.

\section{REFERÊNCIAS}

ALDRICH, C.; FENG, D. The effect of mothers on bubble size distributions in flotation pulp phases and surface froths. Minerals Engineering, v. 13, n. 10, p. 1049-1057, 2000.

CHO, YOON-SEONG; LASKOWSKI, J. S. Effect of flotation frothers on bubble size and foam stability. International Journal of Mineral Processing, v. 64, n. 2, p. 69-80, 2002.

FILHO, A. M. R.; GARDUSI, Fernanda; CARVALHO, Guilherme R. L.; LUZ, Pedro H. M.; REIS, Angelica S.; BARROZO, Marcos A. S. Estudo de técnicas para monitoramento da distribuição do diâmetro de bolhas na flotação, Jorneq, UFU,Uberlândia (MG), 2014.

FINCH, J. A.; NESSET, Jan E.; ACUÑA, Claudio. Role of frother on bubble production and behaviour in flotation. Minerals Engineering, v. 21, n. 12, p. 949-957, 2008.

GRAU, R. A.; LASKOWSKI, Janusz S.; HEISKANEN, Kari. Effect of frothers on bubble size. International Journal of Mineral Processing, v. 76, n. 4, p. 225-233, 2005.

LEIVA, J.; VINNETT, L.; CONTRERAS, F.; YIANATOS, J. Estimation of the actual bubble surface area flux in flotation. Minerals Engineering, v. 23, n. 11, p. 888-894, 2010.

SANTOS, M.A. A flotação por ar dissolvido como alternativa ao tratamento de efluente mineral visando ao reuso da água e à melhoria do processo de flotação de apatita. Tese (Doutorado em Engenharia Química), Universidade Federal de Uberlândia, Uberlândia, MG, 2014

SHUKLA, S.C.; KUNDU, G.; MUKHERJEE, D. Study of gas holdup and pressure characteristics in a column flotation cell using coal. Minerals Engineering, v. 23, n. 8, p. 636-642, 2010.

SOBHY, A.; TAO, D. Nanobubble column flotation of fine coal particles and associated fundamentals. International Journal of Mineral Processing, v. 124, p. 109-116, 2013.

RIBEIRO, J. A. Contribuição ao estudo experimental e em CFD da fluidodinâmica de colunas de bolhas com aplicações na separação de misturas oleosas e processamento mineral por ar dissolvido, Tese de Doutorado, UFU, Uberlândia (MG), 2012.

\section{Agradecimentos}

Agradecimentos especiais à Capes e ao $\mathrm{CNPq}$, como órgãos de fomento da pesquisa e à Faculdade de Engenharia Química da Universidade Federal de Uberlândia pelo incentivo e apoio à realização deste trabalho. 